\title{
ANÁLISE DOS ÍNDICES DE CONFORTO TÉRMICO PMV E PET EM DIFERENTES TIPOS DE COBERTURAS EM AMBIENTE ABERTO NA CIDADE DE CUIABÁ-MT
}

\author{
RIBEIRO, Karyn Ferreira Antunes - karyn.ribeiro@cba.ifmt.edu.br \\ Instituto Federal do Mato Grosso / IFMT \\ JUSTI, Ana Clara Alves - clarasevla@gmail.com \\ Universidade Federal do Mato Grosso / UFMT
}
SANTOS, Flavia Maria de Moura - flavia_mms@hotmail.com
Universidade Federal do Mato Grosso / UFMT
NOGUEIRA, Marta Cristina de Jesus Albuquerque - mcjanp@gmail.com Universidade Federal do Mato Grosso / UFMT

\author{
MUSIS, Carlo Ralph de - carlo.demusis@gmail.com \\ Universidade de Cuiabá / UNIC
}

\begin{abstract}
RESUMO: Objetivou-se com o presente estudo analisar os índices de conforto térmico PMV e PET em diferentes tipos de coberturas em ambiente aberto. Logo, coletou-se variáveis climáticas por meio de estações micro meteorológicas fixas e para estimar o conforto térmico, aplicou-se questionários com perguntas subjetivas e processamento com o software RayMan. A menor variação de temperatura do ar foi verificada no cenário arborizado, com valores de 27,5 a $35,2^{\circ} \mathrm{C}$ no período quente-úmido e de 22,1 a $34,8{ }^{\circ} \mathrm{C}$ no período quente-seco. A umidade relativa do ar, também teve a menor variação neste cenário, com valores de 40,0 a $80,0 \%$ no período quente-úmido e de 28,7 a $81,1 \%$ no período quente-seco. Já a velocidade do vento exerceu pouca influência, com intensidade alternando entre calmo a brisa leve. Os melhores valores de PMV e PET foram expressos no cenário arborizado, com valores que variaram de 0,1 a 3,9 e de $-4,0$ a 3,8 e de 27,5 a $37,4{ }^{\circ} \mathrm{C}$ e de 20,3 a $36,3{ }^{\circ} \mathrm{C}$ para os períodos quente-úmido e quente seco respectivamente. Conclui-se que os índices PMV E PET demonstraram-se eficientes para estimar o conforto térmico, concordando com o comportamento das variáveis climáticas aferidas e ressaltando a importância da arborização nas cidades.
\end{abstract}

PALAVRAS-CHAVE: temperatura do ar, umidade relativa do ar, vegetação.

ANALYSIS OF THE THERMAL COMFORT INDEXES PMV AND PET IN DIFFERENT TYPES OF COVERAGE IN AN OPEN ENVIRONMENT IN THE CITY OF CUIABÁ-MT

\begin{abstract}
The objective of this study was to analyze the thermal comfort indexes PMV and PET in different types of roofing in an open environment. Therefore, climate variables were collected through fixed micro-meteorological stations and to estimate thermal comfort, questionnaires with subjective questions were applied and processing with RayMan software. The smallest variation in air temperature was observed in the wooded scenario, with values of 27.5 to $35.2{ }^{\circ} \mathrm{C}$ in the hot-humid period and 22.1 to $34.8{ }^{\circ} \mathrm{C}$ in the hot-dry period. Relative humidity also had the lowest variation in this scenario, with values from 40.0 to $80.0 \%$ in the hot-humid period and from 28.7 to $81.1 \%$ in the hotdry period. Wind speed, on the other hand, had little influence, with intensity alternating between calm and light breeze. The best PMV and PET values were expressed in the wooded scenario, with values ranging from 0.1 to 3.9 and -4.0 to 3.8 and 27.5 to $37.4{ }^{\circ} \mathrm{C}$ and 20,3 to $36.3{ }^{\circ} \mathrm{C}$ for the hot-humid and hot dry periods respectively. It was concluded that the PMV and PET indices were efficient to estimate the thermal comfort, agreeing with the behavior of the measured climatic variables and emphasizing the importance of afforestation in the cities.
\end{abstract}


KEYWORDS: air temperature, relative humidity, vegetation.

ANÁLISIS DE LOS ÍNDICES DE CONFORT TÉRMICO PMV Y PET EN DIFERENTES TIPOS DE COBERTURA EN UN ENTORNO ABIERTO EN LA CIUDAD DE CUIABÁ-MT

RESUMEN: El objetivo de este estudio fue analizar los índices de confort térmico PMV y PET en diferentes tipos de techos en un entorno abierto. Por lo tanto, las variables climáticas se recolectaron a través de estaciones micro meteorológicas fijas y para estimar el confort térmico, se aplicaron cuestionarios con preguntas subjetivas y se rellenado el software RayMan. La variación más pequeña en la temperatura del aire se observó en el escenario arbolado, con valores de 27.5 a $35.2{ }^{\circ} \mathrm{C}$ en el período de húmedo-caliente y de 22.1 a $34.8^{\circ} \mathrm{C}$ en el período de seco-caliente. La humedad relativa también tuvo la variación más baja en este escenario, con valores del 40.0 al $80.0 \%$ en el período de húmedo-caliente y del 28.7 al $81.1 \%$ en el período de seco-caliente. La velocidad del viento, por otro lado, tuvo poca influencia, con una intensidad alternando entre la brisa tranquila y la ligera. Los mejores valores de PMV y PET se expresaron en el escenario arbolado, con valores que van desde 0.1 a 3.9 y -4.0 a 3.8 y 27.5 a $37.4^{\circ} \mathrm{C}$ y 20,3 a $36.3^{\circ} \mathrm{C}$ para los períodos húmedo-caliente y seco-caliente, respectivamente. Se concluyó que los índices de PMV y PET fueron eficientes para estimar el confort térmico, coincidiendo con el comportamiento de las variables climáticas medidas y enfatizando la importancia de la forestación en las ciudades.

PALABRAS CLAVE: temperatura del aire, humedad relativa del aire, vegetación.

\section{INTRODUÇÃO}

O conforto térmico de espaços abertos tornou-se um tema cada vez mais importante no contexto da tendência mundial de urbanização desenfreada. Isso porque, devido ao efeito de formação da ilha de calor, à poluição do ar e ao declínio das áreas verdes nos recintos urbanos, modificações significativas no clima local podem acontecer (SHARMIN, STEEMERS \& HUMPHREYS, 2019).

Logo, com o aumento das alterações microclimáticas, sérias consequências negativas são percebidas no ambiente, na saúde, na sociedade e na economia. Estas incluem a diminuição da produtividade e desempenho dos trabalhadores, a duração e frequência das atividades ao ar livre (SHARIFI, SIVAM \& BOLAND, 2016), o desenvolvimento de tempestades, o aumento na demanda de energia (SANTAMOURIS et al., 2015; DEILAMI, KAMRUZZAMAN \& HAYES, 2016; SIDIQUI, HUETE \& DEVADAS, 2016), a elevação da intensidade e duração das ondas de calor e consequentemente alterações da umidade relativa do ar (SACHINDRA et al., 2015), além da diminuição da qualidade de vida, saúde, bem-estar e do conforto térmico dos cidadãos (FONG et al. 2019).

Diante desse contexto, muitas técnicas de mitigação são recomendadas para amenizar os impactos das condições microclimáticas. As mais divulgadas e eficientes abrangem o uso de elementos reflexivos em construções (AKBARI \& LEVINSON, 2008; ZHANG et al., 2017) a integração de tecnologia avançada (KARLESSI et al., 2011; XAMAN et al., 2017), a incorporação de resfriamento terrestre e evaporativo (MIHALAKAKOU et al., 1994; ZHANG et al., 2017, a implementação de parques urbanos (SKOULIKA et al. 2014; LIN et al., 2015; ALGECIRAS et al., 2016; JUSTI et al., 2019) e o aumento da vegetação (SANTAMOURIS et al., 2012; BESIR, 2018).

A introdução da vegetação no ambiente urbano é reconhecida como uma solução econômica, baseada na natureza para amenizar as sequelas do aquecimento (TAN \& JIM, 2017). Ela pode ser incorporada na paisagem de uma 
cidade em quatro formas principais (NORTON et al., 2015), ou seja, espaços abertos (LEHMANN et al., 2014), árvores espalhadas (ABREU-HARBICH, LABAKI \& MATZARAKIS, 2015; TAN, LAU \& NG, 2016; TAN, LAU \& NG, 2017), telhados verdes (SHAFIQUE, KIM \& RAFIQ, 2018; TANG \& ZHENG, 2019) e paredes verdes (JIM, 2015; LEE \& JIM, 2017).

As árvores plantadas em espaços abertos a nível de pedestres são particularmente úteis para reduzir o estresse térmico (Sun et al., 2017). Essa característica ocorre, pois, as árvores com copas maiores podem interceptar uma quantidade significativa de radiação solar (SHAHIDAN, 2010), que é o parâmetro meteorológico mais influente no conforto térmico (NG \& CHENG, 2012; COUTTS et al., 2016).

Entretanto, estudos sobre as decorrências do resfriamento da vegetação urbana se concentraram principalmente em árvores e gramíneas (LIN \& LIN, 2010 ;) poucos comparam a vegetação com estruturas de sombreamento artificiais (LEE; HOLST \& MAYER, 2013).

Dessa forma, compreender os efeitos térmicos de diferentes elementos da paisagem é particularmente importante na fase de concepção dos espaços abertos (KONG et al., 2017), tendo em vista que um bom projeto paisagístico que otimize o ambiente térmico, pode melhorar diretamente a sua utilização.

Para tal compreensão, diversos índices de conforto térmico ao ar livre foram criados, baseados nas equações de balanço de energia do corpo humano e nos mecanismos de transferência de calor. Entre eles, os mais populares e amplamente aceitos são o Voto Médio Predito (Predicted Mean Vote - PMV) (FANGER, 1970) e o de Temperatura Fisiológica Equivalente (Physiological Equivalent Temperature - PET), (HÖPPE, 1984).

O PMV permite a predição da sensação térmica de um grupo de pessoas, em uma escala de sete pontos, combinando e considerando distintamente as variáveis temperatura do ar, umidade relativa do ar, velocidade do ar e temperatura radiante média, com o nível de atividade física e vestimenta (ZARE et al., 2018).

Segundo Höppe (1999), o PET é definido como a temperatura fisiológica equivalente, em um ambiente (interno ou externo), à temperatura do ar de um cenário interno de referência, no qual o balanço térmico do corpo humano é mantido com temperatura da pele e do centro do corpo iguais àquelas das condições em avaliação (MARRA, 2017).

Nessa perspectiva, o objetivo da presente pesquisa foi analisar os índices de conforto térmico PMV e PET em diferentes tipos de coberturas em ambiente aberto na cidade de Cuiabá-MT.

\section{MATERIAIS E MÉTODOS}

\section{1 ÁREA DE ESTUDO}

Cuiabá, capital do estado de Mato Grosso, localiza-se na região centrooeste brasileira, à $15^{\circ} 10^{\prime}$ e $15^{\circ} 50^{\prime}$ de latitude sul e $50^{\circ} 50^{\prime}$ e $50^{\circ} 10^{\prime}$ de longitude oeste, com altitude média inferior a $200 \mathrm{~m}$ acima do nível do mar. É conhecida como uma das cidades mais quentes do Brasil e segundo a classificação de Köppen-Geiger, seu perfil climático é tropical continental semiúmido, do tipo Aw, 
com duas estações representadas por chuvas na primavera-verão e por estiagem no outono-inverno.

A temperatura média máxima anual do ar em Cuiabá varia de 31 a $34{ }^{\circ} \mathrm{C}$ e a média mínima anual, varia entre 18 a $21^{\circ} \mathrm{C}$. Ao mesmo tempo que, a umidade relativa do ar tem a média anual de $70 \%$ na estação chuvosa, alcançando valores de $12 \%$ na estação de estiagem (MAITELLI, 2005; ROCHA, 2018). Já a direção do vento é predominante norte e noroeste durante a maior parte do ano, e sul no outono-inverno. Enquanto que, o total anual de precipitação pluviométrica é em torno de $1.350 \mathrm{~mm}$ (INPE, 2018).

Para o presente estudo, escolheu-se uma praça central da Universidade Federal de Mato Grosso (UFMT), que se situa na região leste de Cuiabá, (Figura $1)$.
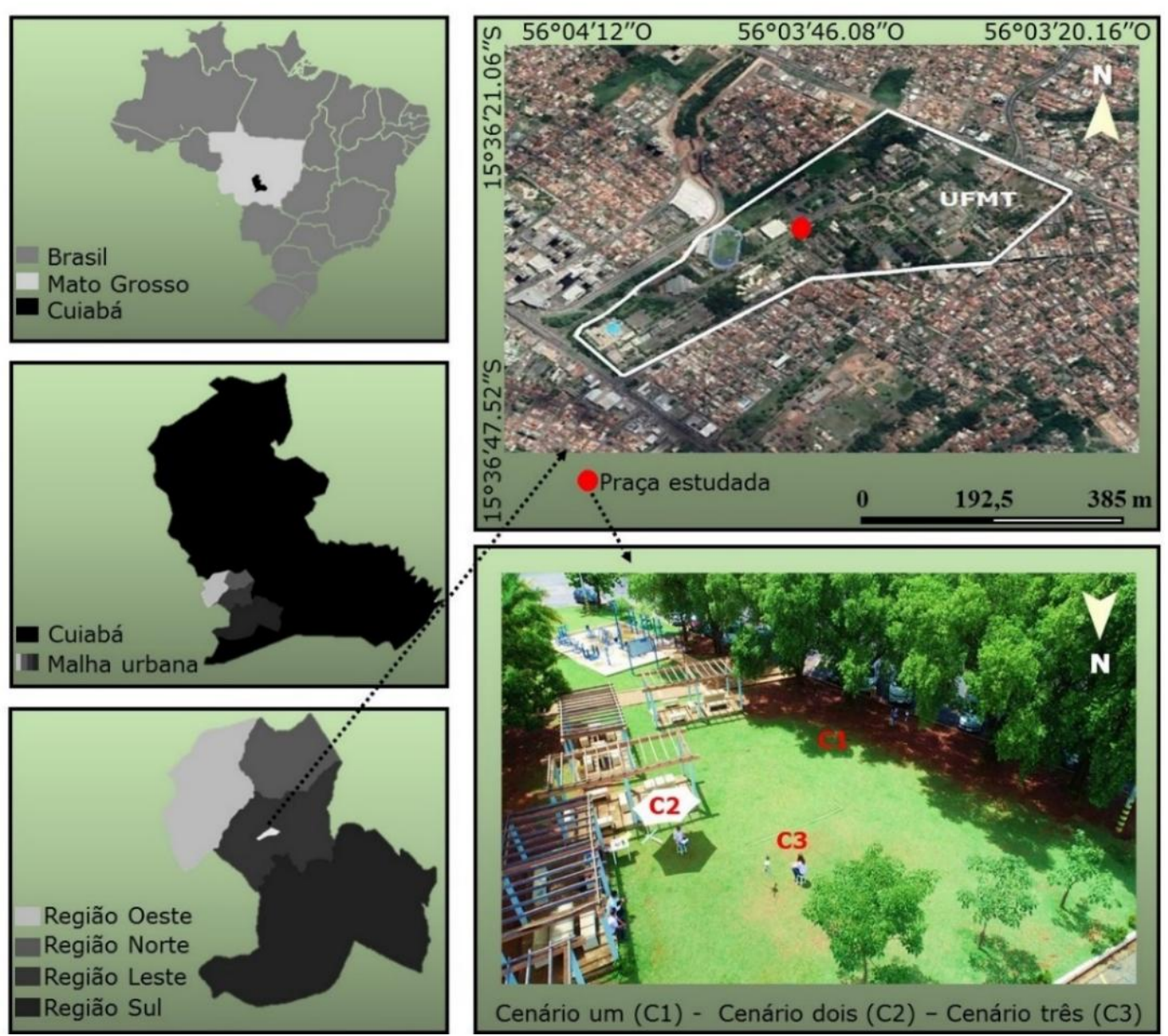

Figura 1 - Localização da área de estudo em relação à UFMT, às regiões e a cidade de Cuiabá, ao estado e ao país. Fonte: Adaptada de Google Earth, 2019

A UFMT detém 74 hectares de área heterogênea, composta por diferentes tipos de uso e ocupação do solo (CAMPOS NETO, 2007), dentre os quais destacam-se as praças, que são locais de áreas verdes utilizados pelos estudantes e visitantes, para a prática de esporte e lazer. 
Desse modo, a escolha do local baseou-se por ser representativo da porção urbana de Cuiabá e pelo próprio ambiente que possibilita a coleta de dados em três cenários distintos. O cenário um (C1) caracterizado por sombreamento arbóreo propiciado pela espécie Licania tomentosa (oiti), o cenário dois (C2) representado por sombreamento artificial de um guarda-sol $100 \%$ poliéster, com $3 \mathrm{~m}$ de diâmetro e $2,45 \mathrm{~m}$ de altura e o cenário três (C3), definido como totalmente exposto ao sol.

\subsection{PROCEDIMENTO EXPERIMENTAL}

Selecionada a área de estudo, o procedimento experimental aconteceu em período integral, com início às $9 \mathrm{h00/8h00} \mathrm{e} \mathrm{término} \mathrm{às} 18 \mathrm{~h} 00 / 17 \mathrm{~h} 00$ do horário normal e de verão respectivamente. Entretanto, por se tratar de um estudo que depende de dias com condições atmosféricas favoráveis, ou seja, ventos fracos e céu com ausência de nuvens (OKE, 1982), foram amostrados somente dois dias típicos de cada estação e período do ano, conforme a Tabela 1 exibe.

Tabela 1 - Datas amostradas

\begin{tabular}{|c|c|c|c|c|}
\hline Mês/Ano & Dias & Horário & Estação & Período \\
\hline Novembro/2018 & 26 & \multirow{2}{*}{$\begin{array}{c}9 \mathrm{~h} 00 \\
\text { às } 18 \mathrm{~h} 00 \\
\end{array}$} & \multirow{2}{*}{ Primavera } & \multirow{4}{*}{ Quente-úmido } \\
\hline Dezembro/2018 & 06 & & & \\
\hline Fevereiro/2019 & $25 *$ & \multirow{2}{*}{$\begin{array}{c}8 \mathrm{~h} 00 \\
\text { às } 17 \mathrm{~h} 00\end{array}$} & \multirow{2}{*}{ Verão } & \\
\hline Março/2019 & $8 * *-18 *-19 * *$ & & & \\
\hline Maio/2019 & 17 & \multirow{2}{*}{$\begin{array}{c}8 \mathrm{~h} 00 \\
\text { às } 17 \mathrm{~h} 00\end{array}$} & \multirow{2}{*}{ Outono } & \multirow{4}{*}{ Quente-seco } \\
\hline Maio/2019 & 29 & & & \\
\hline Julho/2019 & 16 & \multirow{2}{*}{$\begin{array}{c}8 \mathrm{~h} 00 \\
\text { às } 17 \mathrm{~h} 00\end{array}$} & \multirow[t]{2}{*}{ Inverno } & \\
\hline Agosto/2019 & $7 *-15^{* *}$ & & & \\
\hline
\end{tabular}

* Coleta realizada das $8 \mathrm{~h} 00$ às $12 \mathrm{~h} 00$

** Coleta realizada das $13 \mathrm{~h} 00$ às $17 \mathrm{~h} 00$

Os entrevistados foram convidados previamente para participar da pesquisa, seguindo a mesma metodologia adotada por Cheng et al. (2011). O grupo total de participantes foi de 30 pessoas, na sua maioria alunos de graduação, pós-graduação e professores, visto que o local do estudo foi dentro de uma universidade.

O mesmo grupo participou de todas as coletas realizadas, no total foram respondidos 1.134 questionários, sendo 378 questionários por cenário. As porcentagens dos entrevistados do sexo feminino foram de $56,6 \%$ e de $43,4 \%$ para o masculino. Para o cálculo dos índices térmicos PET e PMV foi necessário adotar um participante médio para os diferentes sexos e utilizou-se para o sexo masculino: altura de $1,74 \mathrm{~m}$, peso de $74 \mathrm{~kg}$, e idade 54 anos, e para o sexo feminino: altura de 1,54m, peso de $64 \mathrm{~kg}$ e idade de 24 anos.

Conforme a disponibilidade de cada participante, foi realizada uma escala para o dia e o horário da colaboração na pesquisa. Com objetivo de padronizar a taxa metabólica os voluntários ficaram sentados (atividade metabólica de 55 W, conforme a ISO, 2005) em todos os cenários e utilizaram camisas na cor 
branca $(0,5$ clo, conforme a ISO, 2007) para que não houvesse interferência da roupa na sensação térmica.

O tempo coleta em cada cenário foi de 10 min e aproximando-se do fim do período de 10 min os questionários eram preenchidos e em seguida a troca de cenário era efetuada, sempre em sentido horário. Assim, para o total de 1 hora, os participantes ficaram 2 vezes em cada um dos cenários, conforme mostra a Figura 2-a e b.
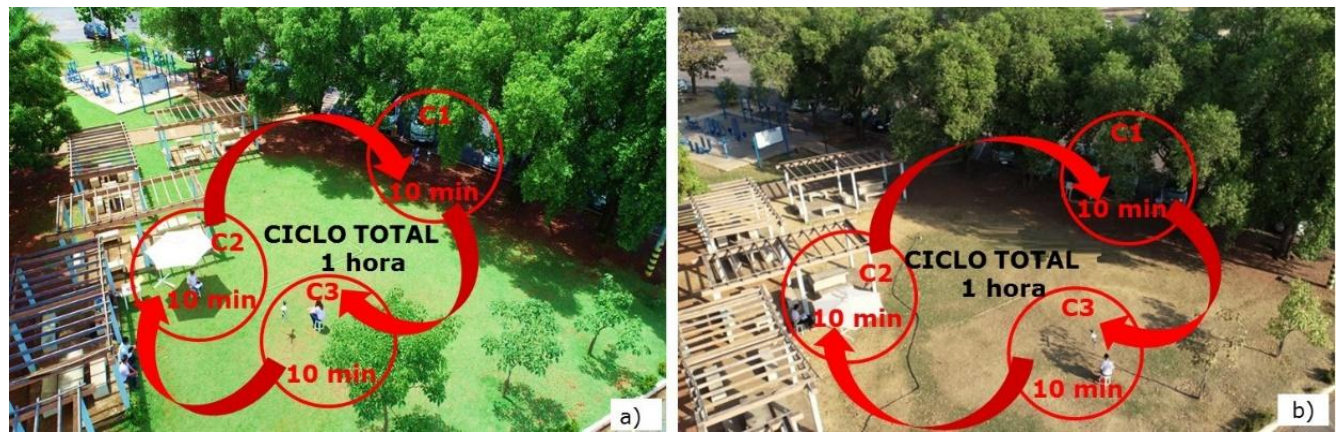

Figura 2 - a) Ciclo realizado no período quente-úmido, b) Ciclo realizado no período quente-seco

Os questionários compreendem perguntas relacionadas à percepção térmica, sensação térmica, preferência térmica, sensibilidade às condições climáticas e características pessoais como: altura, peso, idade, sexo. O mesmo adotado por Hirashima (2010).

Para a coleta de variáveis climáticas, foram posicionadas três estações micro meteorológicas iguais, fixas cada uma em um cenário, a $1 \mathrm{~m}$ de altura da superfície do solo, com sensores dispostos em uma barra de metal de aproximadamente $70 \mathrm{~cm}$, coletando dados de temperatura do ar (Ta), temperatura de globo ( $\mathrm{Tg})$, umidade relativa do ar (UR), direção e velocidade do vento (Vv), (Figura 3).

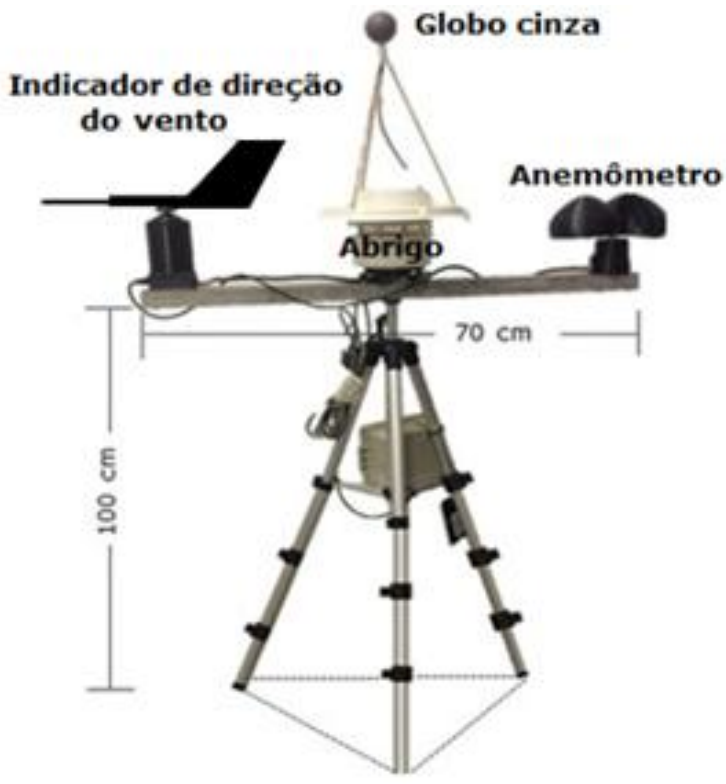

Figura 3 - Representação da estação micrometeorológica utilizada 
É importante mencionar que os globos e os abrigos, foram confeccionados a partir de informações encontradas na literatura, conforme proposto e desenvolvido por Hirashima e Assis (2011), que detalham a confecção e aferição de ambos.

Já a velocidade e direção do vento foram monitoradas por anemômetros de conchas e indicadores de direção do vento, projetados e desenvolvidos pelo grupo de instrumentação científica do Programa de Pós-Graduação em Física Ambiental (PGFA) da UFMT.

As variáveis $\mathrm{Ta}, \mathrm{Tg}$ bem como a UR de cada um dos três cenários, foram registradas por meio de sensores termo higrômetros do tipo datalogger, modelo HOBO U12-012 - Onset, protegidos por abrigos alternativos de Policloreto de Vinila (PVC) e com cabeamento externo acoplados a globos de cor cinza (Figura 4).

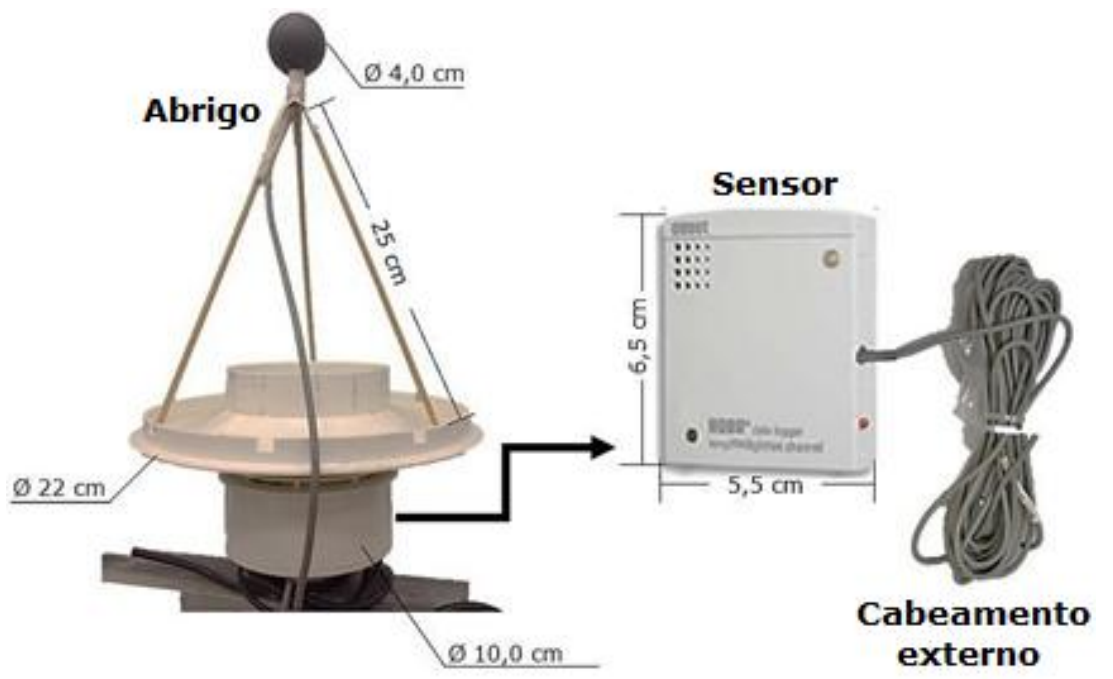

Figura 4 - Representação esquemática do abrigo e do sensor

Nessa perspectiva, as características físicas dos dois sensores são apresentadas na Figura 5- a) e b).
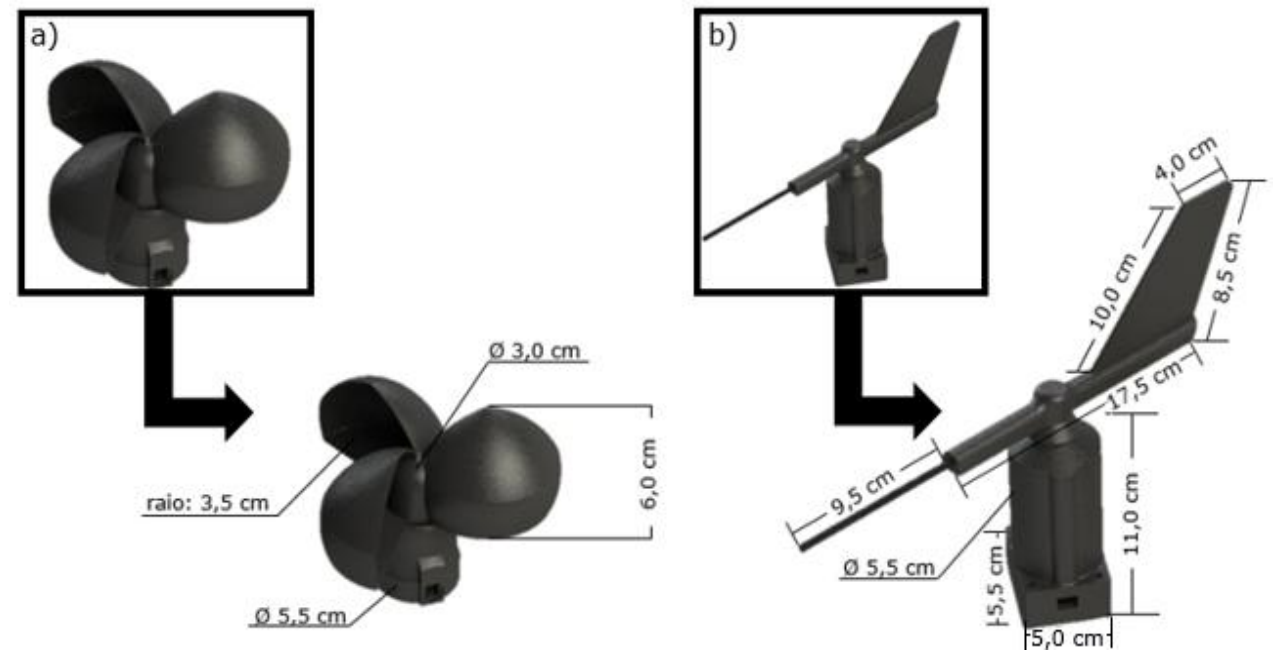

Figura 5 - a) Anemômetro de conchas e b) Indicador de direção do vento 
Para avaliação do desempenho do anemômetro 3D foi realizado o índice de concordância de Wilmott (d), que reflete a precisão dos valores preditos (variável $Y$ ) em relação aos valores observados (variável $X$ ). O índice (d) varia de 0 a 1, quanto mais próximo de 1 significa que a concordância é perfeita, os valores encontrados forma de 0.85 para o $\mathrm{C} 1,0.81$ para o $\mathrm{C} 2$ e 0.78 para o C3.

É válido destacar que apesar de tratar de sensores projetados e desenvolvidos por pesquisadores, mostraram-se eficientes na coleta de dados, servindo como alternativa em meio a limitação de recursos financeiros.

Por fim, para terminar a etapa do procedimento experimental, calculouse a temperatura radiante média (Trm), considerando o diâmetro do globo de 4 $\mathrm{cm}$ e a sua emissividade de 0,9 para a cor cinza, conforme Hirashima e Assis (2011). Foi utilizado a equação da Organização Internacional de Normalização (International Organization for Standardization - ISO) No 7726 de 1998 (ISO, 1998):

$\operatorname{Trm}=\left[\left(T_{g}+273\right)^{4}+\frac{1,1 \cdot 10^{8} \cdot V^{0,6}}{\varepsilon_{g} \cdot D^{0,4}}\left(T_{g}-T_{a}\right)\right]^{\frac{1}{4}}-273$

Em que:

Trm : Temperatura radiante média $\left({ }^{\circ} \mathrm{C}\right)$;

Tg : Temperatura de globo $\left({ }^{\circ} \mathrm{C}\right)$;

Ta : Temperatura do $\operatorname{ar}\left({ }^{\circ} \mathrm{C}\right)$;

V : Velocidade do ar $\left(\mathrm{m} . \mathrm{s}^{-1}\right)$;

$\varepsilon \mathrm{g} \quad$ : Emissividade do globo;

D : Diâmetro do globo (m).

\subsection{ANÁLISE DOS DADOS}

A análise de conforto térmico foi realizada por intermédio dos índices de Voto Médio Predito (Predicted Mean Vote - PMV) e de Temperatura Fisiológica Equivalente (Physiological Equivalent Temperature - PET), determinados através do software RayMan Pro versão 3.1 Beta, desenvolvido por Andreas Matzarakis e disponibilizado de forma gratuita.

Dessa maneira, os dados de entrada foram agrupados com informações referentes à data (dia/mês/ano), hora local $(\mathrm{h})$, temperatura do $\operatorname{ar}\left({ }^{\circ} \mathrm{C}\right)$, umidade relativa do ar (\%), velocidade do vento (m.s-1) e temperatura radiante média $\left({ }^{\circ} \mathrm{C}\right)$ de cada amostragem e cenário. Manualmente completou-se também o banco de dados com informações de localização, latitude, longitude, altitude e fuso horário (UTC+h) locais, além de altura, peso, idade, sexo, vestimenta, atividade metabólica e posição do indivíduo.

Assim, ao término do preenchimento, obteve-se os dados de saída, calculados mediante a seleção dos índices de interesse e para analisar os resultados gerados, utilizou-se a Tabela 2, com calibração para climas tropicais, proposta por Hirashima (2010).

Tabela 2 - Escala de sensação térmica para os índices PMV e PET 


\begin{tabular}{ccc}
\hline PMV & PET & Sensação térmica \\
\hline$-1,5$ a $-3,5$ & sem dados & Muito frio \\
\hline abaixo de $-1,5$ & abaixo de 12,0 & Frio \\
\hline$-1,5$ a $-0,5$ & 12,0 a 15,5 & Pouco frio \\
\hline$-0,5$ a 1,5 & 15,5 a 30,5 & Confortável \\
\hline 1,5 a 2,0 & 30,5 a 31,0 & Pouco quente \\
\hline 2,0 a 3,0 & 31,0 a 35,5 & Quente \\
\hline acima de 3,0 & acima de 35,5 & Muito quente \\
\hline
\end{tabular}

Fonte: Hirashima (2010)

Ademais, os dados de conforto térmico juntamente com os climáticos, passaram também por análise estatística, usufruindo-se do software SPSS versão 16.0 com licença pertencente à Universidade de Cuiabá (UNIC).

Nesse contexto, primeiramente fez-se o bootstrap com 1.000 amostragens e, para a verificação dos pressupostos, homogeneidade, variância e aderência à distribuição normal, utilizou-se o teste de Levene (GLANTZ, 2014; PAULA, 2017; MIRANDA, 2018).

Quando constatado a homocedasticidade dos dados, empregou-se o teste de Tamhane, para analisar e comparar as médias entre os grupos, em relação aos fatores temporais e ou espaciais (MARÔCO, 2018).

\section{RESULTADOS E DISCUSSÃO}

\subsection{ANÁLISE DAS VARIÁVEIS MICROCLIMÁTICAS}

Nos cenários com sombreamento arbóreo (C1), com sombreamento artificial (C2) e totalmente exposto ao sol (C3) a temperatura do ar (Ta) e a umidade relativa do ar (UR) tanto no período quente-úmido, quanto no período quente-seco, apresentaram os comportamentos exibidos na Figura 6-a) e b).

De modo geral, observa-se que independentemente do cenário e período, a faixa de horário das $8 \mathrm{~h} 00$ às $11 \mathrm{~h} 00$ foi a que alcançou os menores valores de Ta e consequentemente os maiores valores de UR. Em contrapartida, das 14h00 às 17 h00 obteve-se os maiores valores de Ta e os menores valores de UR, ressaltando a característica inversamente proporcional de ambas variáveis.

Esse fato pode ser explicado pelo ciclo diurno da Ta, influenciado pela variação da radiação solar. Pois, tipicamente a Ta mais baixa é obtida próxima ao nascer do sol, como resultado de uma noite de resfriamento radiativo da superfície da Terra e a mais alta, usualmente registrada no começo ou meio da tarde, devido ao acúmulo de radiação solar gerado ao longo do dia (ALMEIDA, 2016).

A maior variação de Ta foi aferida no C3, com valores de 27,9 a $37,8{ }^{\circ} \mathrm{C}$ no período quente-úmido e de 21,5 a $36,9{ }^{\circ} \mathrm{C}$ no período quente-seco, representando amplitudes térmicas de $9,9^{\circ} \mathrm{C}$ e $15,4^{\circ} \mathrm{C}$ respectivamente. Neste cenário também se nota a maior variação de UR, com valores de 38,1 a $81,3 \%$ no período quente-úmido e de 26,3 a $81,5 \%$ no período quente-seco, com amplitudes higrométricas equivalentes a 43,2 \% e 55,2 \%. 

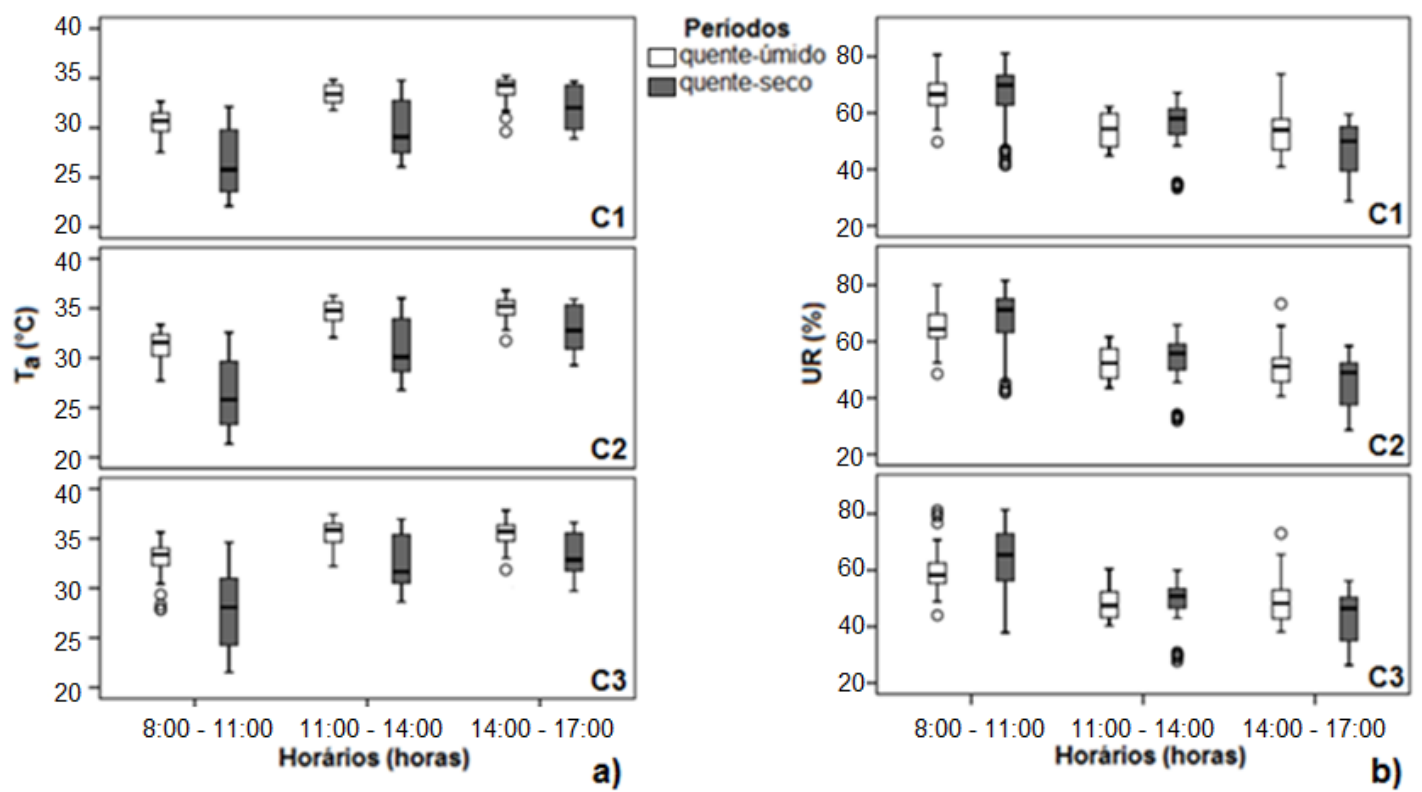

Figura 6 - a) Distribuição da Ta e b) Distribuição da UR

De modo contrário, a menor variação de Ta foi verificada no $\mathrm{C} 1$, com valores de 27,5 a $35,2{ }^{\circ} \mathrm{C}$ no período quente-úmido e de 22,1 a $34,8{ }^{\circ} \mathrm{C}$ no período quente-seco, com amplitudes térmicas de $7,7^{\circ} \mathrm{C}$ e $12,7^{\circ} \mathrm{C}$. Já em relação a UR, a menor variação também foi observada neste cenário, com valores de 40,0 a $80,0 \%$ no período quente-úmido e de 28,7 a $81,1 \%$ no período quente-seco, pontuando amplitudes higrométricas iguais a $40 \%$ e 52,4 $\%$.

Quando comparado com os outros cenários, o C2 foi o que amostrou Ta e UR medianas, com valores de 27,7 a $36,8{ }^{\circ} \mathrm{C}$ e de 40,0 a $80,1 \%$ no período quente-úmido e 21,4 a $36,0{ }^{\circ} \mathrm{C}$ e 28,6 a $81,7 \%$ no período quente-seco, adquirindo amplitudes térmicas de $9,1^{\circ} \mathrm{C}$ e $14,6^{\circ} \mathrm{C}$ e higrométricas de $40,1 \%$ e $53,1 \%$.

Assim, pode-se constatar que a pesquisa em questão representou o contexto micro meteorológico de Cuiabá, uma vez que o período quente-seco obteve Ta menores que o período quente-úmido, principalmente porque entre os meses de maio a agosto, ocorre na região o fenômeno de "friagem" com dias de baixa Ta (SANTOS, 2012). Neste período, a UR também foi característica, pois a sua maior variação é oriunda da transição entre os períodos quente-úmido e quente-seco e posteriormente, em virtude da ausência de chuvas.

Em relação à variável velocidade do vento (Vv), a Figura 7 mostra a distribuição para os três cenários, nos períodos quente-úmido e quente-seco. 


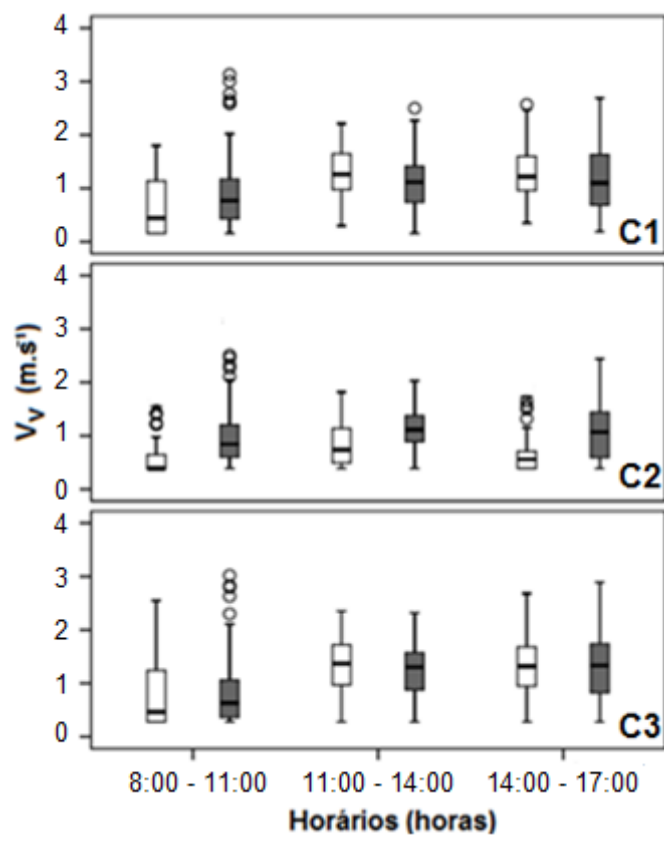

Figura 7 - Velocidade do vento no período quente-úmido e quente-seco

Analisando todos os cenários, percebe-se que a $\mathrm{V} v$ foi praticamente uniforme. No $\mathrm{C} 1$, a variação foi de 0,2 a 2,6 m.s-1 e de 0,2 a 3,1 m.s-1 nos períodos quente-úmido e quente-seco sucessivamente. No C2, de 0,4 a 1,8 m.s1 e de 0,4 a 2,5 m.s-1 e por fim, no C3, de 0,3 a 2,7 m.s-1 e de 0,3 a 3,0 m.s-1 para os mesmos períodos.

De acordo com a Escala Beaufort, a intensidade dos ventos foi de calmo a brisa leve, exercendo pouca influência nas demais variáveis climáticas. Resultados semelhantes também foram encontrados nos trabalhos de (NINCE, 2013; FRANCO, 2013 e FRANÇA, 2017).

\subsection{ANÁLISE DOS ÍNDICES PMV E PET}

Para os índices PMV e PET, os resultados da análise multivariada são apresentados na Tabela 3.

Verifica-se que para o cenário arborizado (C1) e no período quenteúmido, a porcentagem de respostas concentrou-se na sensação térmica equivalente a confortável, com o total de $24,6 \%$, seguida de $7,8 \%$ para pouco quente e de 0,9\% para quente. Enquanto, no período quente-seco, 27,6\% de respostas concentraram-se em confortável, 4,6\% em pouco quente, $0,8 \%$ em pouco frio e $0,3 \%$ em frio.

Diferentemente, para o cenário com sombreamento artificial (C2) e no período quente-úmido, a porcentagem de respostas foi mais expressiva para a sensação térmica correspondente a pouco quente, com o total de $16,7 \%$, seguida de 9,3\% para confortável, 6,3\% para quente e de 1,1\% para muito quente. Já em relação ao período quente-seco, 15,2 \% respostas concentraramse em confortável, $12,6 \%$ em pouco quente, $4,4 \%$ em quente, $0,8 \%$ em pouco frio, $0,2 \%$ em frio e $0,2 \%$ em muito quente. 
Tabela 3 - Qualificação e quantificação das respostas dos questionários

\begin{tabular}{|c|c|c|c|c|c|c|c|}
\hline \multirow{3}{*}{$\begin{array}{l}\text { Sens. } \\
\text { térmica }\end{array}$} & \multirow{3}{*}{ Cenário } & \multicolumn{6}{|c|}{$\begin{array}{c}\text { Períodos } \\
\end{array}$} \\
\hline & & \multicolumn{3}{|c|}{ Quente-úmido } & \multicolumn{3}{|c|}{ Quente-seco } \\
\hline & & $\begin{array}{l}\text { Med. } \\
\text { PMV }\end{array}$ & $\begin{array}{l}\text { Med. } \\
\text { PET }\end{array}$ & $\begin{array}{c}\text { Porc. } \\
\% \\
\end{array}$ & $\begin{array}{l}\text { Med. } \\
\text { PMV }\end{array}$ & $\begin{array}{l}\text { Med. } \\
\text { PET }\end{array}$ & $\begin{array}{c}\text { Porc. } \\
\% \\
\end{array}$ \\
\hline \multirow{3}{*}{ MF } & 1 &. & . & 0 &. &. & 0 \\
\hline & 2 & . & . & 0 & . & . & 0 \\
\hline & 3 & . & . & 0 & . & . & 0 \\
\hline \multirow{3}{*}{$\mathrm{F}$} & 1 & . & . & 0 & $-2,9$ & 22,8 & 0,3 \\
\hline & 2 & . & . & 0 & $-4,4$ & 19,3 & 0,2 \\
\hline & 3 & . & . & 0 & $-3,4$ & 21,0 & 0,2 \\
\hline \multirow{3}{*}{ PF } & 1 & . & . & 0 & $-3,7$ & 20,9 & 0,8 \\
\hline & 2 & . & . & 0 & $-3,5$ & 21,4 & 0,8 \\
\hline & 3 &. & . & 0 & $-2,2$ & 23,1 & 0,8 \\
\hline \multirow{3}{*}{$\mathrm{C}$} & 1 & 2,8 & 33,9 & 24,6 & 0,9 & 29,0 & 27,6 \\
\hline & 2 & 2,8 & 34,2 & 9,3 & 1,1 & 29,4 & 15,2 \\
\hline & 3 & 3,0 & 34,8 & 2,0 & 1,4 & 30,8 & 5,7 \\
\hline \multirow{3}{*}{$P Q$} & 1 & 2,8 & 33,9 & 7,8 & 2,4 & 33,1 & 4,6 \\
\hline & 2 & 3,6 & 36,6 & 16,7 & 2,4 & 33,5 & 12,6 \\
\hline & 3 & 4,2 & 39,4 & 6,9 & 2,7 & 34,5 & 11,1 \\
\hline \multirow{3}{*}{$\mathrm{Q}$} & 1 & 3,1 & 34,3 & 0,9 &. &. & 0 \\
\hline & 2 & 4,2 & 38,4 & 6,3 & 3,7 & 36,7 & 4,4 \\
\hline & 3 & 5,0 & 40,6 & 12,4 & 4,7 & 39,6 & 11,8 \\
\hline \multirow{3}{*}{$M Q$} & 1 &. &. & 0 &. & . & 0 \\
\hline & 2 & 4,3 & 37,6 & 1,1 & 2,8 & 33,9 & 0,2 \\
\hline & 3 & 5,8 & 42,7 & 12,4 & 5,3 & 42,2 & 3,7 \\
\hline
\end{tabular}

Sensação (Sens.) - Mediana (Med.) - Porcentagem (Porc.) - Muito frio (MF) - Frio (F) Pouco Frio (PF) - Confortável (C) - Pouco Frio (PF) - Quente (Q) - Muito Quente (MQ)

Os piores resultados de sensação térmica foram expressos para o cenário totalmente exposto ao sol (C3), que no período quente-úmido, teve a porcentagem de respostas concentradas em muito quente, com o total de 12,4 $\%$, seguida de $12,4 \%$ em quente, $6,9 \%$ em pouco quente e 2,0\% em confortável. Entretanto no período quente-seco, os valores alteraram-se para $11,8 \%$ em quente, $11,1 \%$ em pouco quente, $5,7 \%$ em confortável, 3,7 \% em muito quente, $0,8 \%$ pouco frio e $0,2 \%$ em pouco frio.

Com intuito de melhorar o entendimento, a Figura 8-a) e b) exibem os resultados do índice PMV e PET.

A faixa de muito frio, não aparece na visualização gráfica exatamente por não representar a sensação térmica dos participantes que estavam alocados em cada um dos cenários analisados, para ambos os períodos e índices.

O cenário 1 registrou os melhores valores de PMV, que variaram de 0,1 a 3,9 e de $-4,0$ a 3,8 e com valores de PET que variaram de 27,5 a $37,4{ }^{\circ} \mathrm{C}$ e de 20,3 a $36,3^{\circ} \mathrm{C}$ para os períodos quente-úmido e quente seco respectivamente. 

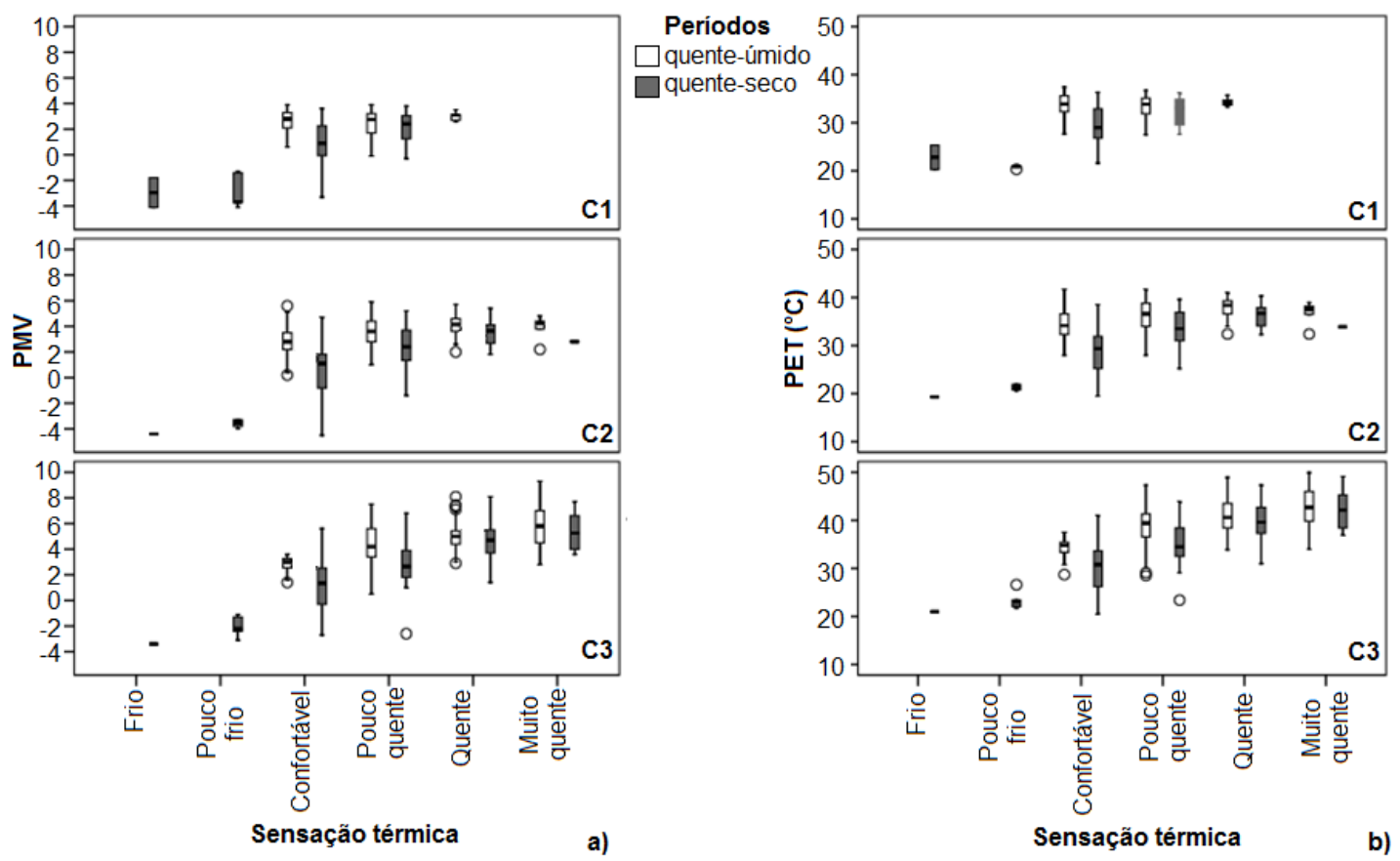

Figura 8 - a) Valores do índice PMV e b) PET no período quente-úmido e quente-seco

Em segundo lugar, destaca-se o cenário 2, com valores de PMV, que variaram de 0,2 a 5,9 e de $-4,5$ a 5,4 e com valores de PET que variaram de 28,0 a $41,7{ }^{\circ} \mathrm{C}$ e de 19,3 a $40,3{ }^{\circ} \mathrm{C}$ para os períodos quente-úmido e quente seco consecutivamente.

O cenário 3 fica em último lugar, com valores de PMV, que variaram de 0,5 a 9,3 e de $-3,1$ a 8,1 e com valores de PET que variaram de 21,0 a $49,9{ }^{\circ} \mathrm{C}$ e de 20,5 a $49,1{ }^{\circ} \mathrm{C}$ para os períodos quente-úmido e quente seco seguidamente, confirmando o comportamento alcançado também pelas variáveis climáticas.

Além disso, ressalta-se a importância da vegetação, pois mesmo com o sombreamento propiciado por um guarda-sol, é no sombreamento provocado pela arborização que os resultados se mostram mais favoráveis ao conforto térmico dos participantes da pesquisa. Isso porque, as árvores absorvem a radiação solar em função de seus processos vitais de fotossíntese e evapotranspiração, e refletem, protegendo efetivamente as superfícies do contato direto de energia solar incidente (DECLET-BARRETO et al., 2016).

\section{CONCLUSÕES}

Os índices PMV E PET demonstraram-se eficientes para estimar o conforto térmico dos ambientes abertos analisados, concordando com 0 comportamento das variáveis climáticas aferidas.

Tanto para o PMV, quanto para o PET, os melhores valores de conforto térmico foram registrados para o cenário com sombreamento arbóreo. Logo, cabe ressaltar a importância da arborização em meio urbano, para melhorar termicamente o ambiente, não só a sensação térmica dos indivíduos, mas 
também no microclima como um todo, essencialmente em cidades que possuem um rigor climático igual ao de Cuiabá.

\section{REFERÊNCIAS}

ABREU-HARBICH, L. V.; LABAKI, L. C. \& MATZARAKIS, A. Effect of tree planting design and tree species on human thermal comfort in the tropics. Landscape and Urban Planning, v. 138, p. 99-109, 2015.

AKBARI, H. \& LEVINSON, R. Evolução dos padrões de telhado frio nos EUA. Advances in Building Energy Research, v. 2, n. 1, p. 1-32, 2008.

ALGECIRAS, J. A. R.; CONSUEGRA, L. G. \& MATZARAKIS, A. Spatial-temporal study on the effects of urban street configurations on human thermal comfort in the world heritage city of Camagüey-Cuba. Building and Environment, v. 101, p. 85-101, 2016.

ALMEIDA, H. A. Climatologia Aplicada à Geografia. Paraíba: Eduepb, 2016. p. 1329.

BESIR, A. B. \& CUCE, E. Green roofs and facades: A comprehensive review, v. 82 n. 1, p. 915-939, 2018.

CAMPOS NETO, A. A. Estudo bioclimático no campus da Universidade Federal de Mato Grosso. 2007. 177f. Dissertação (Mestrado em Física Ambiental), Instituto de Física, Universidade Federal de Mato Grosso, Cuiabá, 2007.

CHENG, V., NG, E., CHAN, C., GIVONI, B. Outdoor thermal comfort study in a subtropical climate: a longitudinal study based in Hong Kong. Int. $\mathrm{J}$. Biometeorol. V. 56, p. 43-56, 2011.

COUTTS, A. M.; WHITE, E. C.; TAPPER, N. J.; BERINGER, J.; LIVESLEY, S. J. Temperature and human thermal comfort effects of street trees across three contrasting street canyon environments. Theoretical and Applied Climatology, v.124, n. 1-2, p. 55-68, 2016.

DECLET-BARRETO, J.; KNOWLTON, K.; JENERETTE, G. D. \& BUYANTUEV, A. Effects of urban vegetation on mitigating exposure of vulnerable populations to excessive heat in Cleveland, Ohio. American Meteorological Society, out. 2016. Disponível em: < http://dx.doi.org/10.1175/WCAS-D-15-0026.s1>. Acesso em: 24 out. 2018.

DEILAMI, K.; KAMRUZZAMAN, M. \& HAYES, J. F. Correlation or causality between land cover patterns and the urban heat island effect? Evidence from Brisbane, Australia. Remote Sensing, v. 8, n. 9, p. 716, 2016.

FANGER, P. O. Thermal comfort. Analysis and application in environment engineering Danish Technical Press. Copenhagen, 1970.

FONG, C. S.; AGHAMOHAMMADI, N.; RAMAKRESHNAN, L.; NIK, M. S. \& MOHAMMADI, P. Holistic recommendations for future outdoor thermal comfort assessment in tropical Southeast Asia: A critical appraisal. Sustainable Cities and Society, v. 46, p. 1-20, 2019.

FRANÇA, M. S. Avaliação do microclima urbano para a cidade de Sorriso/MT: estudo de caso (Doutorado em Física Ambiental) - Instituto de Física da Universidade Federal de Mato Grosso, Cuiabá, p. 1-91, 2017. 
FRANCO, F. M. Análise do comportamento termohigrométrico urbano sob a ótica do uso e ocupação do solo em Cuiabá - MT (Doutorado em Física Ambiental) Instituto de Física da Universidade Federal de Mato Grosso, Cuiabá, p. 1-142, 2013.

GLANTZ, S.A. Princípios de Bioestatística. 7aed. Porto Alegre: Artmed, 2014, p.306.

HIRASHIMA, S. Q. S. Calibração do Índice de conforto Térmico Temperatura Fisiológica Equivalente (PET) para Espaços Abertos do Município de Belo Horizonte, MG. 2010. 225f. Dissertação (Mestrado Insterdisciplinar em Ambiente Construído e Patrimônio Sustentável), Universidade Federal de Minas Gerais, Belo Horizonte, 2010.

HIRASHIMA, S. Q. S. \& ASSIS, E. S. Confecção e aferição de termômetro de globo e abrigo meteorológico para medição de variáveis climáticas em ambientes externos. XI Encontro Nacional de Conforto no Ambiente Construído ENCAC, Búzios, RJ, 10 f., 2011.

HÖPPE, P. Die Energiebilanz des Menschen. München Universitäts Schriften, Fachbereich Physic. Wissenschaftliche Mitteilungen, p. 49, 1984.

HÖPPE, P. R. The physiological equivalent temperature: a universal index for the biometeorological assessment of the thermal environment. International Journal of Biometeorology, 43, p. 71- 75, 1999.

INPE - Instituto Nacional de Pesquisas Espaciais. 2018. Disponível em: <http://sonda.ccst.inpe.br/estacoes/cuiaba_clima.html>. Acesso em: 14 de junho de 2019.

INTERNATTIONAL ORGANIZATION DOR STANDARDIZATION ISO 7726: Ergonomics of the thermal environment -- instruments for measuring physical quantities. Genève: ISO, 1998.

INTERNATTIONAL ORGANIZATION DOR STANDARDIZATION ISO 9920: Ergonomics of thermal environments - Estimation of the thermal insulation and water vapor resistance of a clothing ensemble. Genève: ISO, 2007.

INTERNATTIONAL ORGANIZATION DOR STANDARDIZATION ISO 7730: Modarate thermal environments - Determination of the PMV and PPD indices and specification of the conditions for thermal comfort. Genève: ISO, 2005.

JIM, C. Y. Thermal performance of climber greenwalls: Effects of solar irradiance and orientation. Applied Energy, v. 154, p. 631-643, 2015.

JUSTI, A. C. A.; NOGUEIRA, M. C. J. A.; SANTOS, F. M. M.; DE MUSIS, C. R. \& NOGUEIRA, J. S. Impacto da morfologia de parque urbano no microclima e no conforto térmico de Cuiabá-Brasil. Revista Brasileira de Climatologia, v. 24, p. 20-38, 2019.

KALESSI, T.; SANTAMOURIS, M.; SYNNEFA, A.; ASSIMAKOPOULOS, D. DIDASKALOPOULOS P. \& APOSTOLAKIS, K. Development and testing of PCM doped cool colored coatings to mitigate urban heat island and cool buildings Building and Environment, v. 46 n. 3, p. 570-576, 2011.

KONG, L.; LAU, K. K.; YUAN, C.; CHEN, Y.; XU, Y.; REN, C. \& NG, E. Regulation of outdoor thermal comfort by trees in Hong Kong. Sustainable Cities and Society, v. 31, p. 12-25, 2017. 
LEE, H.; HOLST, J. \& MAYER, H. Modification of Human-Biometeorologically Significant Radiant Flux Densities by Shading as Local Method to Mitigate Heat Stress in Summer within Urban Street Canyons. Advances in Meteorology, v. 2013, p. 1-13, 2013.

LEE, L. S. H. \& JIM, C. Y. Subtropical summer thermal effects of wirerope climber green walls with different air-gap depths. Building and Environment, $v$. 126, p. 1-12, 2017.

LEHMANN, I.; MATHEY, J.; ROBLER, S.; BRAUER, A. \& GOLDBERG, V. Urban vegetation structure types as a methodological approach for identifying ecosystem services - Application to the analysis of micro-climatic effects. Ecological Indicators, v. 42, p. 58-72, 2014.

LIN, B. S. \& LIN, Y. J. Cooling effect of shade trees with different characteristics in a subtropical urban park. HortScience, v. 45, p. 83-86, 2010.

LIN, W.; YU, T.; CHANG, X.; WU, W. \& ZHANG, Y. Calculating cooling extents of green parks using remote sensing: Method and test. Landscape and Urban Planning, v. 134, p. 66-75, 2015.

MAITELLI, G. T. Interações Atmosfera-Superfície. In: Moreno G.; Higa T. C. S. organizadores. Geografia de Mato Grosso: território, sociedade e ambiente. Entrelinhas: Cuiabá, p. 1-296, 2005.

MARÔCO, J. Análise Estatística com o SPSS Statistics. 7aed. Report Number, Lda. 2018, p.650.

MARRA, N. C. S. N. Avaliação do conforto térmico em conjunto habitacional de interesse social-Simulação computacional com o programa Solene Microclima. 2017. 147 f. Dissertação (Mestrado em Ambiente Construído e Patrimônio Sustentável) - Escola de Arquitetura, Universidade Federal de Minas Gerais, Belo Horizonte, 2017.

MIHALAKAKOU, G.; SANTAMOURIS, M. \& ASIMAKOPOULOS, D. On the cooling potential of earth to air heat exchangers. Energy Conversion and Management, v. 35, n. 5, p. 395-402, 1994.

MIRANDA, S. A. Sombreamento arbóreo em superfícies pavimentadas de área urbana tropical. 2018. 91 f. Tese (Doutorado em Física Ambiental) - Instituto de Física, Universidade Federal de Mato Grosso, Cuiabá, 2018.

NG, E. \& CHENG, E. Urban human thermal comfort in hot and humid Hong Kong. Energy and Buildings, v. 55, p. 51-65, 2012.

NINCE, P. C. d O C. Vegetação e revestimentos urbanos: implicações na sensação térmica dos usuários do campus da UFMT em Cuiabá-MT (Doutorado em Física Ambiental) - Instituto de Física da Universidade Federal de Mato Grosso, Cuiabá, p. 1-108, 2013.

NORTON, B. A. ; COUTTS, A. M.; LIVESLEY, J.; HARRIS, R. J.; HUNTER, A. M. \& WILLIAMS, N. S. G. Planning for cooler cities: A framework to prioritise green infrastructure to mitigate high temperatures in urban landscapes. Landscape and Urban Planning, v. 134, p. 127-138, 2015.

OKE, T.R. The energetic basis of the urban heat island (Symons memorial lecture, 20 May 1980). Quarterly Journal of the Royal Meteorological Society, v.108, n. 455, pp. 1-24, 1982. 
PAULA, D. C. J. Análise termohigrométrica pós intervenções urbana em CuiabáMT. 2017. 90 f. Dissertação (Mestrado em Física Ambiental) - Instituto de Física, Universidade Federal de Mato Grosso, Cuiabá, 2017.

ROCHA, A. D. da. Espectro cruzado e coerência wavelet: um estudo de variáveis micrometeorológicas em frações urbanas na cidade de Cuiabá, MT. 2018. 142f. Tese (Doutorado em Física Ambiental), Instituto de Física, Universidade Federal de Mato Grosso, Cuiabá, 2018.

SACHINDRA, D. A.; NG, S. W. M.; MUTHUKUMARAN, S. \& PERERA, B. J. C. Impact of climate change on urban heat island effect and extreme temperatures: a case-study. Quarterly, Journal of the Royal Meteorological Society, v. 142, p. 172-186, 2015.

SANTAMOURIS, M.; CARTALIS, C.; SYNNEFA, A. \& KOLOKOTSA, D. On the impact of urban heat island and global warming on the power demand and electricity consumption of buildings - A review. Energy Buildings, v. 98, p. 119124, 2015.

SANTAMOURIS, M.; XIRAFI, F.; GAITANI, N.; SPANOU, A.; SALIARI, M. \& VASSILAKOPOULOU, $\mathrm{K}$. Improving the microclimate in a dense urban area using experimental and theoretical techniques - The case of Marousi, Athens International Journal of Ventilation, v. 11, n. 1, p. 1-16, 2012.

SANTOS, F. M. de M. Influência da ocupação do solo na variação termohigrométrica na cidade de Cuiabá-MT (Doutorado em Física Ambiental) Instituto de Física da Universidade Federal de Mato Grosso, Cuiabá, p. 1-88, 2012.

SHAFIQUE, M.; KIM, R. \& RAFIQ, M. Green roof benefits, opportunities and challenges - A review. Renewable and Sustainable Energy Reviews, v. 90, p. 757-773, 2018.

SHAHIDAN, M. F.; SHARIFF, M. K. M.; JONES, P.; SALLEH, E. \& ABDULLAH, A. $M$. A comparison of Mesua ferrea $L$. and Hura crepitans L. for shade creation and radiation modification in improving thermal comfort. Landscape and Urban Planning, v. 97, n. 3, p. 168-181, 2010.

SHARIFI, E.; SIVAM, A. \& BOLAND, J. Resilience to heat in public space: a case study of Adelaide, South Australia. Journal of Environmental Planning and Management, v. 59, n.10, p. 1833-1854, 2016.

SHARMIN, T.; STEEMERS, K. \& HUMPHREYS, M. Outdoor thermal comfort and summer PET range: A field study in tropical city Dhaka. Energy and Buildings, $v$. 198, p. 149-159, 2019.

SIDIQUI, P.; HUETE, A. \& DEVADAS, R. Spatio-temporal mapping and monitoring of Urban Heat Island patterns over Sydney, Australia using MODIS and Landsat-8. Sensing applications (EORSA). In: 4th International Workshop on, p. 217-221, 2016.

SKOULIKA, F.; SANTAMOURIS, M.; KOLOKOTSA, D. \& BOEMI, N. On the thermal characteristics and the mitigation potential of a medium size urban park in Athens, Greece. Landscape and Urban Planning, v. 123, p. 73-86, 2014.

SUN, S.; XU, X.; LAO, Z.; LIU, W.; LI, Z.; GARCÍA, E. H.; HE, L. \& ZHU, J. Evaluating the impact of urban green space and landscape design parameters on 
thermal comfort in hot summer by numerical simulation. Building and Environment, v. 123, p. 277-288, 2017.

TAN, P. Y. \& JIM, C. Y. Urban greening and microclimate modification. Greening Cities, p. 73-93, 2017.

TAN, Z.; LAU, K. K. \& NG, E. Urban tree design approaches for mitigating daytime urban heat island effects in a high-density urban environment. Energy and Buildings, v. 114, p. 265-274, 2016.

TAN, Z.; LAU, K. K. \& NG, E. Planning strategies for roadside tree planting and outdoor comfort enhancement in subtropical high-density urban areas. Building and Environment, v. 120, p. 93-109, 2017.

TANG, M. \& ZHENG, X. Experimental study of the thermal performance of an extensive green roof on sunny summer days. Applied Energy, v. 242, p. 1010$1021,2019$.

XAMAN, J.; CISNEROS-CARREÑO, J.; HERNÁNDEZ-PÉREZ, I.; HERNÁNDEZLOPEZ, I.; AGUILAR-CASTRO, K. M. \& MACIAS-MELO, E. V. Thermal perfomance of a hollow block with/without insulating and reflective materials for roofing in Mexico. Applied Thermal Engineering, v. 123, p. 243-255, 2017.

ZARE, S.; HASHEMINEZHAD, N.; SAREBANZADEH, K.; ZOLALA, F.; HEMMATJO, R. \& HASSANVAND, D. Assessing thermal comfort in tourist attractions through objective and subjective procedures based on ISO 7730 standard: a field study. Urban Climate, v. 26, p. 1-9, 2018.

ZHANG, L.; MENG, X.; LIU, F.; XU, L. \& LONG, E. Effect of retro-reflective materials on temperature environment in tents. Case Studies in Thermal Engineering, v. 9, p. 122-127, 2017.

ZHANG, Y.; ZHANG, L.; PAN, Z.; MENG, Q.; FENG, Y. \& CHEN, Y. Hydrological properties and solar evapirative cooling performance of porous clay tiles. Construction and Building Materials, v. 151, p. 9-17, 2017 